\title{
Schizophreniform Psychosis at Onset of Adrenoleukodystrophy
}

\section{To the Editor:}

Psychotic symptoms were described at multiple neurological diseases. Their presentation with neurological signs or findings at image modalities, should conduce to a complex process of differential diagnosis. X-linked adrenoleukodystrophy (ALD) shows a wide range of phenotypic variability. The following case report presents a patient with schizophreniform psychosis at adult onset of ALD.

\section{CASE REPORT}

A 43-year-old female admitted to the psychiatric hospitalization ward with severe psychotic symptomatology is presented. Confined to a bed for the last three months, her physical and nutritional state were very bad. She had poor academic achievement. Her sister presented a severe mental deficiency and her mother mild learning difficulties. Psychiatric treatment onset for behavioral disturbances was at 25 years of age, and 4 years later she was hospitalized, receiving a diagnosis of dissociative disorder. At current admission patient was conscious, with negative attitude and akinetic mutism that alternated with agitation episodes, echolalia and palilalia; auditory hallucinations presented as an only voice which she identifies like her boyfriend that control her behavior; erotomaniac delusions; decrease of the intake; and insomnia. Anamnesis revealed a poor functional level among both hospital incomes.

On neurologic examination the absence of reflex and marked loss of position sense to passive movement in the legs were found. Neuropsychological examination, performed using subtest of the Barcelona Programa integrado de exploración neuropsicológica Test neuropsychological battery, Rey complex figure and Rey Auditory Verbal Learning Test, resulted in progressive impairment on a previ- ous deficiency (memory deficits, abstract thinking, learning processes and visuoconstructive tasks, and executive dysfunction with impaired mental control, thinking flexibility and planning).

She presented borderline low concentration of vitamin B12 in the serum $(217 \mathrm{pg} / \mathrm{dL})$ and erithrocyte sedimentation rate was persistent high. A lumbar puncture disclosed a cerebrospinal fluid with a high protein $1.80 \mathrm{~g} / \mathrm{L}$, but normal cell count and glucose. Electrophysiological examination showed an axon loss motor and sensory polyneuropathy. Electroencephalography and visual and auditory potentials did not present pathological data. Cortical atrophy mainly frontal was found by brain computed tomography. Symmetrical hyperintensities in the parieto-occipital regions were detected on T2-weighted and flair magnetic resonance imaging (Figure). Sural nerve biopsy revealed mixed

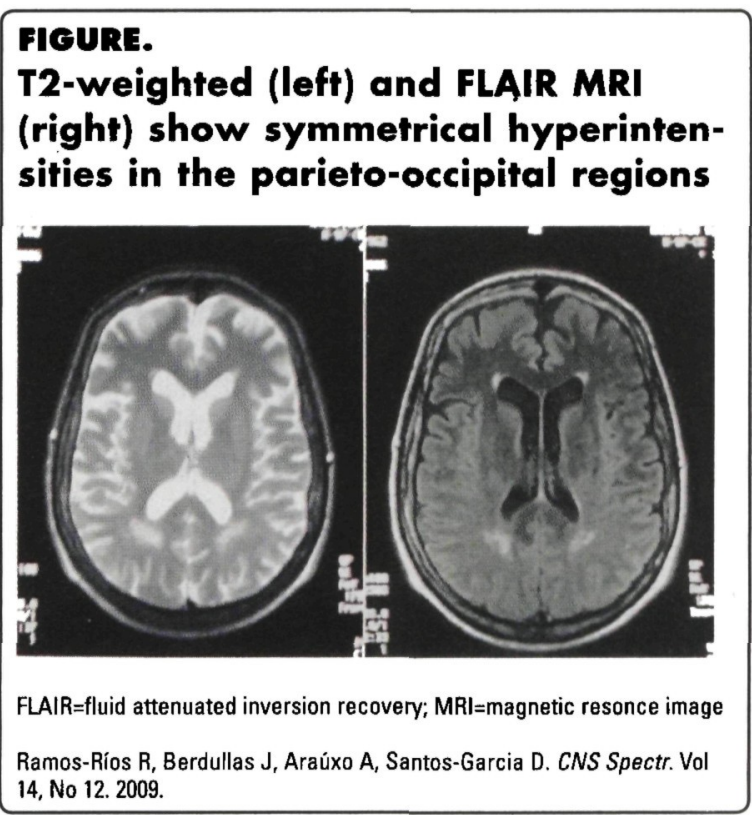


demyelination and remyelination with axonal degeneration. Finally increased concentrations of saturated very long chain fatty acids (VLCFAs) in blood confirmed the diagnosis of ALD. VLCFAs were elevated as follows: $\mathrm{C} 26: 0$ was $3 \mathrm{umol} / \mathrm{L}$ (normal range $0.55 \pm 0.17$, the ratio of $\mathrm{C} 24: 0 / \mathrm{C} 22: 0$ was $0.91 \mathrm{umol} / \mathrm{L}(0.77 \pm 0.12)$ and the ratio of $\mathrm{C} 26: 0 / \mathrm{C} 22: 0$ was $0.063 \mathrm{umol} / \mathrm{L}(0.012 \pm 0.004)$.

She was initially treated with haloperidol $30 \mathrm{mg} /$ day with a partial response (with worst response to risperidone and aripiprazole). Catatonic symptoms required ECT ( 8 sessions) with a moderate improvement of the psychotic features, but persisting cognitive impairment. Subsequently the patient was admitted to a chronic unit of a psychiatric hospital.

\section{DISCUSSION}

ALD is an inherited disease characterized by the accumulation of VLCFAs in plasma and several tissues: brain, testis, adrenal cortex, or skin. In the nervous system, VLCFA tended to accumulate in myelin-producing cells (both peripheral and central). This abnormality could be produced due to a peroxisomal dysfunction with an incomplete degradation of VLCFA. These are described in three forms: the classic or childhood-onset form, an adolescent onset form with a rapid progress, and an adult onset form with a more variable course. The adult onset form shows a wide range of phenotypic variability. ${ }^{1}$ It could present like an adrenocortical insufficiency with myelopathy and neuropathy (adrenomyeloneuropathy) or with predominant central nervous system affectation and psychiatric symptomatology, including schizophreniform psychosis and mania, which progress to dementia. ${ }^{2-6}$ Although it is considered an $\mathrm{x}$-linked disease, as much as $50 \%$ of female heterozygotes were reported to present several neurological signs and symptoms. These patients usually have a làter onset, better prognosis and no adrenal dysfunction.?

The presence of a non-responsive psychiatric illness and an abnormal magnetic resonance image, typically with T2-weighted symmetrical hyperinten- sities in the parieto-occipital regions, should make the clinician suspect this kind of metabolic disease. The definitive diagnosis of ALD is made by means of the measurement of VLCFA in serum. There is no effective treatment, although dietary treatment (Lorenzo's oil), immunosuppressive therapies, and bone marrow transplantation have been proposed. However, there is consensus about the difficulty of treatmenting the psychiatric complications that frequently appear during the course of the illness. ${ }^{8}$

$X$-linked adrenoleukodystrophy (X-ALD) is probably underdiagnosed as many clinicians are not aware of the relatively high incidence and the clinical presentations of the different phenotypes of $\mathrm{X}$-ALD, including psychiatric symptomatology. Not only men are affected; female carriers are at risk for developing several symptoms as well. This case illustrates the importance of performing neurologic and radiological examinations on all psychiatric patients who are not responding to standard treatment. The clinical problem of being "treatment resistant" should be seen as an indication that other diagnoses need to be considered.

\author{
Sincerely, \\ Ramón Ramos-Ríos, MD \\ José Berdullas, PsyD \\ Alberte Araúxo, MD \\ Diego Santos-García MD, PhD
}

\section{REFERENCES}

1. Moser HW, Raymond GV, Dubey P. Adrenoleukodistrophy, new approaches to a neurodegenerative disease. JAMA. 2005;294:3131-3134.

2. Rosebush PI, Garside S, Levinson AJ, Mazurek MF. The neuropsychiatry of adultonset adrenoleukodystrophy. J Neuropsychiatry Clin Neurosci. 1999:11:315-327.

3. Kopala LC, Tan S. Shea C. Orlik H, Vandorpe R, Honer WG. Adrenoleukodystrophy associated with psychosis. Schizophr Res. 2000;45:263-265.

4. Menza MA, Blake J, Goldberg L. Affective symptoms and adrenoleukodystrophy: A report of two cases. Psychosomatics. 1988;29:442-445.

5. James ACD, Kaplan P. Lees A, Bradley JJ. Schizophreniform psychosis and adrenomyeloneuropathy. Journal of the Royal Society of Medicine. 1984;77:882-884.

6. Angus B, de Silva R, Davidson R, Bone I. A family with adult-onset cerebral adrenoleukodystrophy. J Neurol. 1994;241:497-499.

7. Moser HW. Adrenoleukodystrophy: phenotype, genetics, pathogenesis and therapy. Brain. 1997:120:1485-1508.

8. Garside S, Rosebush Pl, Levinson AJ, Mazurek MF. Late-onset adrenoleukodystrophy associated with long-standing psychiatric symptoms. J Clin Psychiatry. 1999;60:460-468.

\footnotetext{
Dr. Ramos-Ríos is resident of Psychiatry at the Complexo Hospitalario Universitario de Santiago. Dr. Araúxo is psychiatrist and associate professor in the Department of Psychiatry at the Complexo Hospitalario Universitario de Santiago. When the authors participated in the attention and diagnostic process of the patient, J. Berdullas was resident of Psychology and Dr. Santos-García was resident of Neurology at the Complexo Hospitalario Universitario de Santiago.

Faculty Disclosures: The authors do not have an affiliation with or financial interest in any organization that might pose a conflict of interest. Submitted for publication: July 23, 2008; Accepted for publication: August 14, 2009.
} 


\section{NO PASSWORD. NO FEE. NO HASSLE. JUST PEER-REVIEWED CLINICAL INFORMATION WWW.cnsspectrums.com}
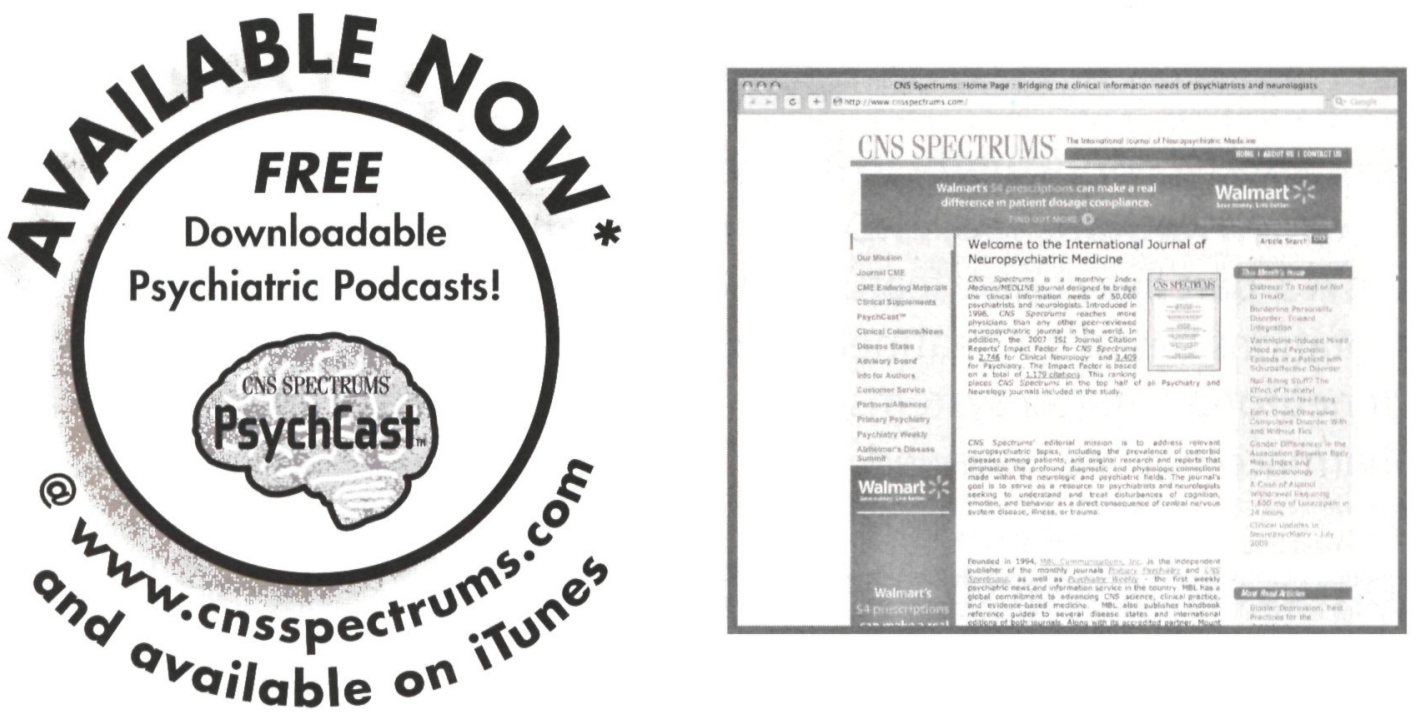

"Click on the PsychCast ${ }^{\text {TM }}$ button at www.cnsspectrums.com

CNS-Based Podcast Programming from the Publishers of Primary Psychiatry, CNS Spectrums, and Psychiatry Weekly

CNS Spectrums' Web portal is now better than ever - a one-stop source providing the following integrated services based on input from you... our readers:

- eSubmissions of Manuscripts and ePeer Review via ScholarOne's Manuscript Central

- RSS Feeds for Current Issues, CME articles and Academic Supplements

- Enhanced Citation-based Article Search

- Dynamic Pop-Up Article Images

- Downloadable Article PDFs

- elearning via Enduring Materials
- Most-Read Articles automatically tabulated

- Integrated Customer Service Tools, including an Online Store featuring all Clinical Handbooks

- A host of additional services and features:...including hyperlink access to MBL's other CNS sources:

www.primarypsychiatry.com and www.psychiofryweekly.com

To learn more, please visit www.cnsspectrums.com or $\mathbf{w w}$ w.mblcommunications.com PrIMARY PSYCHATRYY CNS SPECTRUMS PsychiatryWeekly. 


\section{Stahl's}

\section{Essential Psychopharmacology Masters Course}

A Curriculum-Driven Comprehensive Survey of the Field in Three Semesters

A unique educational opportunity to network with high-level colleagues and the instructor, Stephen M. Stahl, MD, PhD, author of Stahl's Essential Psychopharmacology, Third Edition! A 3-part live course featuring concepts from this latest best-seller and theoretical presentations based on treatment and outcomes from Dr. Stahl's own practice. Seven major neurotransmitter systems, seven major drug classes and ten critical concepts of psychopharmacology are reviewed.

\section{Each semester is worth up to 15.0 AMA PRA Category 1 Credits $^{\mathrm{TM}}$}

\section{REGISTER TODAY! FOR 1, 2 OR ALL 3 SEMESTERS}

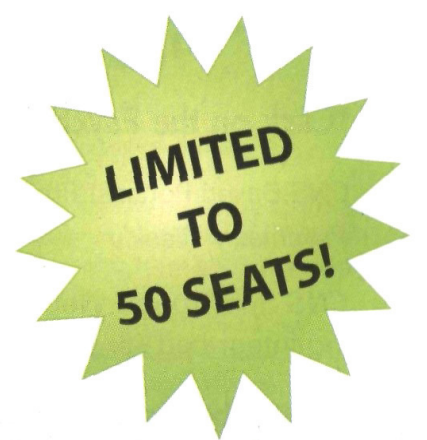

\section{Course Dates and Topics:}

July 17-19, 2009

Semester One:

Psychosis and Antipsychotics
October 9-11, 2009

Semester Two:

Mood, Pain, Antidepressants and Mood Stabilizers
January 15-17, 2010

Semester Three:

Special Topics--Anxiety, Drug Abuse,

Sleep/Wake, ADHD and Dementia

Registration Options:

Phone: $+1(888) 535-5600$

Fax: $+1(760) 931-8713$

Online: www.neiglobal.com

The Neuroscience Education Institute is accredited by the Accreditation Council for Continuing Medical Education to provide continuing medical education for physicians. 


\section{INTUNIV ${ }^{\mathrm{TM}}$ (guanfacine) Extended-Release Tablets}

\section{Rx Only}

BRIEF SUMMARY: Consult the Full Prescribing Information for complete product information.

\section{INDICATIONS AND USAGE}

INTUNIVTM is indicated for the treatment of Attention Deficit Hyperactivity Disorder (ADHD). The efficacy of INTUNIVTM was studied for the treatment of ADHD in two controlled clinical trials (8 and 9 weeks in duration) in children and adolescents ages 6-17 who met DSM-IV ${ }^{\circledR}$ criteria for ADHD (see Clinical Studies in Full Prescribing Information). The effectiveness of INTUNIVTM for longer-term use (more than 9 weeks) has not been systematically evaluated in controlled trials.

Maintenance Treatment The effectiveness of INTUNIVTM for longer-term use (more than 9 weeks) has not been systematically evaluated in controlled trials. Therefore the physician electing to use INTUNIV ${ }^{T M}$ for extended periods should periodically re-evaluate the long-term usefulness of the drug for the individual patient.

\section{CONTRAINDICATIONS}

Patients with a history of hypersensitivity to INTUNIVTM, its inactive ingredients (see Description in Full Prescribing Information), or other products containing guanfacine (e.g. TENEX®) should not take INTUNIV'M.

\section{WARNINGS AND PRECAUTIONS}

Hypotension, Bradycardia, and Syncope Treatment with INTUNIVTM can cause decreases in blood pressure and heart rate. In the pediatric, short-term (8-9 weeks), controlled trials, the maximum mean changes from baseline in systolic blood pressure, diastolic blood pressure, and pulse were $-5 \mathrm{~mm} \mathrm{Hg},-3 \mathrm{~mm} \mathrm{Hg}$, and $-6 \mathrm{bpm}$, respectively, for all dose groups combined (generally one week after reaching target doses of $1 \mathrm{mg} /$ day, $2 \mathrm{mg} /$ day, $3 \mathrm{mg} /$ day or $4 \mathrm{mg} /$ day). These changes were dose dependent. Decreases in blood pressure and heart rate were usually modest and asymptomatic; however, hypotension and bradycardia can occur. Hypotension was reported as an adverse event for $6 \%$ of the INTUNIVIM group and $4 \%$ of the placebo group. Orthostatic hypotension was reported for $1 \%$ of the INTUNIV ${ }^{\mathrm{M}}$ group and none in the placebo group. In long-term, open label studies, (mean exposure of approximately 10 months), maximum decreases in systolic and diastolic blood pressure occurred in the first month of therapy. Decreases were less pronounced over time. Syncope occurred in $1 \%$ of pediatric subjects in the clinical program. The majority of these cases occurred in the long-term, open-label studies. Measure heart rate and blood pressure prior to initiation of therapy, following dose increases, and periodically while on therapy. Use INTUNIVTM with caution in patients with a history of hypotension, heart block, bradycardia, or cardiovascular disease, because it can decrease blood pressure and heart rate. Use caution in treating patients who have a history of syncope or may have a condition that predisposes them to syncope, such as hypotension, orthostatic hypotension, bradycardia, or dehydration. Use INTUNIVIM with caution in patients treated concomitantly with antihypertensives or other drugs that can reduce blood pressure or heart rate or increase the risk of syncope. Advise patients to avoid becoming dehydrated or overheated.

Sedation and Somnolence Somnolence and sedation were commonly reported adverse reactions in clinical studies (38\% for INTUNIVTM vs. $12 \%$ for placebo) in children and adolescents with ADHD, especially during initial use (see Adverse Reactions in Full Prescribing Information). Before using INTUNIVTM with other centrally active depressants (such as phenothiazines, barbiturates, or benzodiazepines), consider the potential for additive sedative effects. Caution patients against operating heavy equipment or driving until they know how they respond to treatment with INTUNIVIM. Advise patients to avoid use with alcohol.

Other Guanfacine-Containing Products Guanfacine, the active ingredient in INTUNIV ${ }^{M}$, is also approved as an antihypertensive. Do not use INTUNIV'M in patients concomitantly taking other guanfacine-containing products (e.g., Tenex).

\section{ADVERSE REACTIONS}

Clinical Trial Experience Two short-term, placebo-controlled, double-blind pivotal studies (Studies 1 and 2) were conducted in children and adolescents with ADHD with a dose range of 1 to $4 \mathrm{mg} /$ day of INTUNIVTM. The most commonly reported adverse reactions (occurring in $\geq 2 \%$ of patients) that were considered drug-related and reported in a greater percentage of patients taking INTUNIV ${ }^{\mathrm{TM}}$ compared to patients taking placebo were: somnolence, headache, fatigue, upper abdominal pain, nausea, lethargy, dizziness, irritability, hypotension/decreased blood pressure, decreased appetite, dry mouth, and constipation. Less common adverse reactions $(<2 \%)$ reported in pivotal Studies 1 and 2 that occurred in more than one patient taking INTUNIV ${ }^{\mathrm{M}}$ and were more common than in the placebo group are atrioventricular block, bradycardia, sinus arrhythmia, dyspepsia, asthenia, chest pain, increased alanine aminotransferase, increased blood pressure, increased weight, postural dizziness, increased urinary frequency, enuresis, asthma, orthostatic hypotension, and pallor. In addition, the following less common $(<2 \%)$ psychiatric disorders occurred in more than one patient receiving INTUNIV V'M and were more common than in the placebo group. The relationship to INTUNIVTM could not be determined because these events may also occur as symptoms in pediatric patients with ADHD: agitation, anxiety, depression, emotional lability, nightmares or interrupted sleep. Twelve percent $(12 \%)$ of patients receiving INTUNIVTM discontinued from the clinical studies due to adverse events, compared to $4 \%$ in the placebo group. The most common adverse reactions leading to discontinuation of INTUNIVTM-treated patients from the studies were somnolence/sedation $(6 \%)$ and fatigue $(2 \%)$. Less common adverse reactions leading to discontinuation (occurring in approximately $1 \%$ of patients) included: hypotension/decreased blood pressure, headache, and dizziness. In the controlled long term studies (mean duration of approximately 10 months) with a dose range of 1 to $4 \mathrm{mg} /$ day of INTUNIV ${ }^{\mathrm{M}}$, the most common adverse reactions $(\geq 5 \%)$ reported during open label treatment were somnolence, headache, fatigue, upper abdominal pain, hypotension/decreased blood pressure, vomiting, dizziness, nausea, weight increased, and irritability. The most frequent adverse reactions leading to discontinuation $(\geq 2 \%)$ were somnolence $(3 \%)$, syncopal events $(2 \%)$, increased weight $(2 \%)$, depression $(2 \%)$, and fatigue $(2 \%)$. Other adverse reactions leading to discontinuation in the long-term studies (occurring in approximately $1 \%$ of patients) included: hypotension/decreased blood pressure, sedation, headache, and lethargy. In long-term open label studies, serious adverse reactions occurring in more than one patient were syncope $(2 \%)$ and convulsion $(0.4 \%)$. Adverse reactions that occurred in $<5 \%$ of patients but $\geq 2 \%$ in open-label, long-term studies that are considered possibly related to INTUNIVTM include: syncopal events, constipation, stomach discomfort, hypertension/increased blood pressure, decreased appetite, diarrhea, dry mouth, lethargy, and insomnia.

Effects on Height, Weight, and Body Mass Index (BMI) Patients taking INTUNIVTM demonstrated similar growth compared to normative data. Patients taking INTUNIVTM had a mean increase in weight of $1 \mathrm{~kg}(2 \mathrm{lbs})$ compared to those receiving placebo over a comparative treatment period. Patients receiving INTUNIV ${ }^{T M}$ for at least 12 months in open-label studies gained an average of $8 \mathrm{~kg}(17 \mathrm{lbs})$ in weight and $8 \mathrm{~cm}(3 \mathrm{in})$ in height. The height, weight, and BMI percentile remained stable in patients at 12 months in the long-term studies compared to when they began receiving INTUNIV ${ }^{\mathrm{M}}$.

Laboratory Tests In short and long-term studies, no clinically important effects were identified on any laboratory parameters.

Effects on Heart Rate and QT Interval The effect of two dose levels of immediaterelease guanfacine ( $4 \mathrm{mg}$ and $8 \mathrm{mg}$ ) on the QT interval was evaluated in a doubleblind, randomized, placebo- and active-controlled, cross-over study in healthy adults. A dose-dependent decrease in heart rate was observed during the first 12 hours, at time of maximal concentrations. The mean change in heart rate was $-13 \mathrm{bpm}$ at $4 \mathrm{mg}$ and $-22 \mathrm{bpm}$ at $8 \mathrm{mg}$. An apparent increase in mean QTC was observed for both doses. However, guanfacine does not appear to interfere with cardiac repolarization of the form associated with pro-arrhythmic drugs. This finding has no known clinical relevance.

\section{USE IN SPECIFIC POPULATIONS}

Pregnancy: Pregnancy Category B. There are no adequate and well-controlled studies of guanfacine in pregnant women. This drug should be used during pregnancy only if clearly needed.

Nursing Mothers: It is not known whether guanfacine is excreted in human milk. Because many drugs are excreted in human milk, caution should be exercised when INTUNIVTM is administered to a nursing woman.

Pediatric Use: The safety and efficacy of INTUNIVTM in pediatric patients less than 6 years of age have not been established.

Geriatric Use: The safety and efficacy of INTUNIV ${ }^{\mathrm{M}}$ in geriatric patients have not been established.

\section{DRUG ABUSE AND DEPENDENCE}

INTUNIVTM is not a controlled substance and has no known potential for abuse or dependence.

\section{OVERDOSAGE}

Two cases of accidental overdose of INTUNIV'M were reported in clinical trials in pediatric ADHD patients. These reports included adverse reactions of sedation and bradycardia in one patient and somnolence and dizziness in the other patient. Consult with a Certified Poison Control Center for up to date guidance and advice.

Manufactured for Shire US Inc., Wayne, PA 19087.

INTUNIVTM is a trademark of Shire LLC.

(C) 2009 Shire Pharmaceuticals Inc.

August $2009 \quad 5130207001 \quad$ INT-00239 